(A) Check for updates

Cite this: Org. Biomol. Chem., 2021, 19, 1404

Received 15th December 2020,

Accepted 17th January 2021

DOI: 10.1039/d0ob02502j

rsc.li/obc

\section{Synthesis of macrocyclic and medium-sized ring thiolactones via the ring expansion of lactams $\uparrow$}

\author{
Kleopas Y. Palate, (D) Ryan G. Epton, Adrian C. Whitwood, (D) Jason M. Lynam (D) and \\ William P. Unsworth iD *
}

\section{Introduction}

Thioesters are amongst the most important class of small molecules in biology, with thioesters such as acetyl coenzyme A playing central roles in countless biosynthetic processes. ${ }^{1}$ The biochemistry of thioesters has also inspired the development of methods in synthetic chemistry, for example in the native chemical ligation of peptides and during protein splicing. ${ }^{2}$ Thiolactones (the cyclic analogues of thioesters, Fig. 1) also have important biological functions; for example, homocysteine thiolactone $\mathbf{1}$ is involved in the post translational modification of proteins, ${ }^{3 a}$ acts as an allosteric Dopamine D2 receptor antagonist ${ }^{3 b}$ and may have played a role in the development of life on Earth. ${ }^{3 c}$ Thiolactones are also relevant in medicinal chemistry; e.g. as antibiotics (thiolactomycin 2), or as pro-drugs, where the relative ease with which they undergo hydrolysis ${ }^{4}$ is important in revealing the bioactive form in vivo (e.g. 3 and 4). ${ }^{5}$ Indeed, the ability of thiolactones to undergo ring-opening via reaction with water, and other nucleophiles, is often key to their use as reagents in synthetic chemistry ${ }^{6}$ and in polymer science. ${ }^{7}$

Macrocyclic and medium-sized ring thiolactones are relatively rare in the literature. Of those that are known, most are prepared via the condensation of an activated linear thiol-tethered carboxylic acid derivative via an end-to-end cyclisation reaction $\left(\mathbf{5} \rightarrow \mathbf{6}\right.$, Scheme 1a). ${ }^{8}$ As is common for larger ring cyclisation processes of this type, ${ }^{9,10}$ these methods are often low yielding and usually require high dilution conditions and/or slow addition of reagents to afford the thiolactone products. ${ }^{8}$

Department of Chemistry, University of York, Heslington, York, YO10 5DD, UK. E-mail:william.unsworth@york.ac.uk

$\dagger$ Electronic supplementary information (ESI) available. CCDC 1921223, 2040346 and 2040347. For ESI and crystallographic data in CIF or other electronic format see DOI: $10.1039 /$ d0ob02502j
Ring expansion represents an attractive alternative strategy for macrocycle/medium sized ring synthesis, as the inefficient end-to-end cyclisation step can be replaced with a more kinetically favourable rearrangement reaction. ${ }^{11,12}$ However, published ring expansion approaches to make macrocyclic and medium-sized ring thiolactones are limited to only a handful of examples. ${ }^{13,14}$ To the best of our knowledge, the first was reported by Mahajan and Araújo in 1978, and is based on the oxidative cleavage of cyclic vinyl sulfides 7 to form ring expanded thiolactones 8 (Scheme $1 \mathrm{~b}$ ). ${ }^{13 a, b}$ In addition to two papers on the synthesis of medium-sized thiolactones via sigmatropic rearrangements, ${ }^{13 c, d}$ the work most closely related to the present study was reported by Zhang and co-workers in 2014 (Scheme 1c). ${ }^{13 e}$ This study was based on classical side chain insertion of thiol-tethered cyclic nitro ketone 9 a to form thiolactone 10, using a method conceptually related to previous works by Hesse and co-workers. ${ }^{15}$

Our main contribution to the ring expansion field is through the development of an iterative ring expansion strategy known as Successive Ring Expansion (SuRE) ${ }^{16}$ As is illustrated in Scheme 2d, a key aspect of SuRE reactions is that the motif present in the starting material (e.g. the lactam in 11) is regenerated upon ring expansion $(\mathbf{1 1} \rightarrow \mathbf{1 3}),{ }^{16 c, d}$ thus enabling additional iterations of the same reaction to be performed to expand the ring further (e.g. 2 more iterations enable the conversion of $\mathbf{1 3} \rightarrow \mathbf{1 4}$ ). To date, our research has focused on

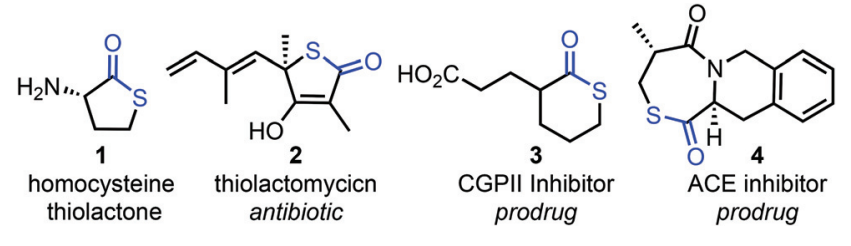

Fig. 1 Biologically important thiolactones 1-4. 


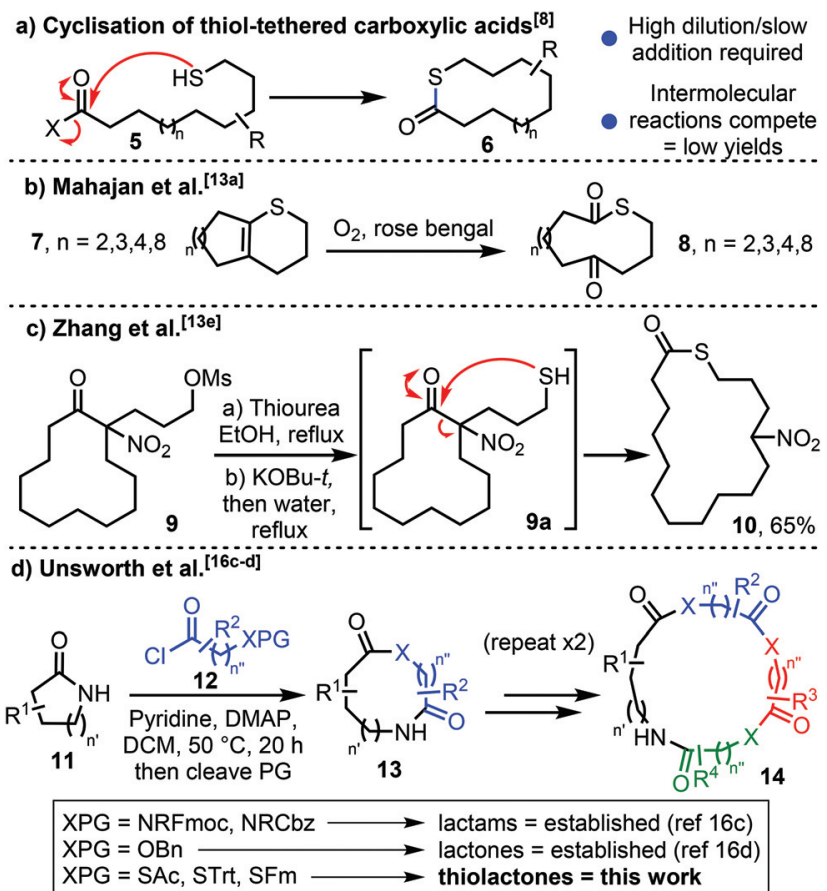

Scheme 1 Strategies to make medium-sized and macrocyclic thiolactones. (a) End-to-end cyclisation; (b) ring expansion via oxidative cleavage; (c) ring expansion via side-chain insertion; (d) successive ring expansion (SURE).

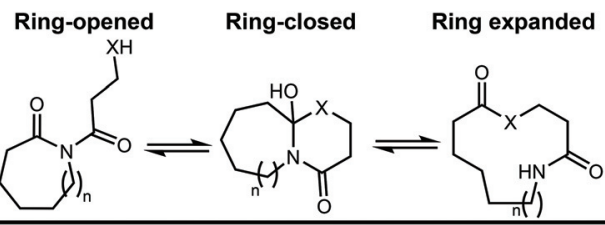

\begin{tabular}{|c|c|c|c|}
\hline $\mathrm{n}=1, \mathrm{X}=\mathrm{S}$ & $15_{\mathrm{Ro}}, \Delta \mathrm{G}^{\circ}$ rel $=0.0$ & $15_{\mathrm{Rc}}, \Delta \mathrm{G}^{\circ} \mathrm{rel}=17.1$ & $15_{\mathrm{RE}}, \Delta \mathrm{G}^{\circ} \mathrm{rel}=3.8$ \\
\hline $\mathrm{n}=1, \mathrm{X}=\mathrm{NH}$ & $16_{\mathrm{RO}}, \Delta \mathrm{G}^{\circ} \mathrm{rel}=0.0$ & $16_{\mathrm{RC}}, \Delta \mathrm{G}^{\circ} \mathrm{rel}=12.1$ & $16_{\mathrm{RE}}, \Delta \mathrm{G}^{\circ}$ rel $=-8.1$ \\
\hline$n=1, X=N M e$ & $17_{\mathrm{RO}}, \Delta \mathrm{G}^{\circ}$ rel $=0.0$ & $17_{\mathrm{RC}}, \Delta \mathrm{G}^{\circ}$ rel $=15.0$ & $17_{\mathrm{RE}}, \Delta \mathrm{G}^{\circ}$ rel $=-5.6$ \\
\hline$n=1, x=0$ & $18_{\mathrm{RO}}, \Delta \mathrm{G}^{\circ} \mathrm{rel}=0.0$ & $1_{\mathrm{RC}}, \Delta \mathrm{G}^{\circ} \mathrm{rel}=9.1$ & $18_{\mathrm{RE}}, \Delta \mathrm{G}^{\circ}$ rel $=-6.9$ \\
\hline$n=0, x=s$ & $19_{\mathrm{RO}}, \Delta \mathrm{G}^{\circ} \mathrm{rel}=0.0$ & $19_{\mathrm{RC}}, \Delta \mathrm{G}^{\circ} \mathrm{rel}=15.4$ & $19_{\mathrm{RE}}, \Delta \mathrm{G}^{\circ} \mathrm{rel}=7.5$ \\
\hline$n=2, X=S$ & $\mathbf{2 0}_{\mathrm{RO}}, \Delta \mathrm{G}^{\circ}$ rel $=0.0$ & $20_{\mathrm{RC}}, \Delta \mathrm{G}^{\circ}$ rel $=20.0$ & $20_{\mathrm{RE}}, \Delta \mathrm{G}^{\circ} \mathrm{rel}=-1.8$ \\
\hline $\mathrm{n}=3, \mathrm{X}=\mathrm{S}$ & $\mathbf{2 1}_{\mathrm{RO}}, \Delta \mathrm{G}^{\circ}$ rel $=0.0$ & $21_{\mathrm{RC}}, \Delta \mathrm{G}^{\circ}$ rel $=17.5$ & $\mathbf{2 1}_{\mathrm{RE}}, \Delta \mathrm{G}^{\circ}$ rel $=-7.1$ \\
\hline
\end{tabular}

Scheme 2 Relative energies of isomeric species in SuRE-type rearrangement of imides using a DFT/B3LYP/6-31G* approach. $\Delta G_{\text {rel }}^{\circ}$ values at $298 \mathrm{~K}$ are given in $\mathrm{kcal} \mathrm{mol}^{-1}$.

SuRE reactions based on the use of amino acid (12, where XPG $=$ NRFmoc or $\mathrm{NRCbz})^{13 c}$ or hydroxy acid $(\mathbf{1 2}$, where XPG = $\mathrm{OBn})^{13 d}$ derivatives, to form ring expanded lactams and lactones respectively. In this manuscript, we describe our efforts to extend this concept to include S-nucleophiles. For various reasons outlined herein, these reactions were considerably more challenging to develop than the analogous processes involving $\mathrm{N}$ - and O-nucleophiles. Nonetheless, the successful synthesis of macrocyclic and medium-sized thiolactones via the ring expansion of lactams with thiol-tethered carboxylic acid derivatives has been achieved and is described here for the first time.

\section{Results and discussion}

\section{Computational chemistry}

In our earlier work, we shied away from using thiol derivatives in SuRE, as we thought the reactions would be less thermodynamically favourable than analogous reactions with amino/ hydroxy acids (and hence might be harder to develop). This notion was based on a consideration of the ring expansion as an equilibrium of the type depicted in Scheme 2; we reasoned that there would be a lower thermodynamic driving force for ring expansion based on the formation of a thioester (when $\mathrm{X}$ $=\mathrm{S}$ ) than there is for the formation of comparatively more stable lactams/lactones (when $\mathrm{X}=\mathrm{NR}$ or $\mathrm{O}$ ), which benefit from greater resonance stabilisation. Indeed, this idea is supported by DFT studies; using a computational method recently established by our groups to assess the viability of SuRE-type reactions, ${ }^{17,18}$ the relative free energies isomers $\mathbf{1 5}_{\mathbf{R O}}, \mathbf{1 5}_{\mathbf{R C}}$, $\mathbf{1 5}_{\mathbf{R E}}$ were calculated at the DFT/B3LYP/6-31G* level for a potential $7 \rightarrow$ 11-membered ring expansion to form $\mathbf{1 5}_{\mathbf{R E}}$. The calculations give a clear steer that this reaction is unlikely to proceed, as the ring opened isomer $\mathbf{1 5}_{\mathbf{R O}}$ was calculated to be lower in energy than the ring expanded isomer $\mathbf{1 5}_{\mathbf{R E}}$ by a significant margin $\left(3.8 \mathrm{kcal} \mathrm{mol}^{-1}\right) .{ }^{19}$ This is in contrast with calculations for the analogous $7 \rightarrow$ 11-membered ring expansion reactions based on lactam and lactone formation (16-18), where the ring expanded isomers were calculated to be lower in energy in each case; indeed, these reactions have been shown to work well in our previous synthetic work. ${ }^{16 c, d}$

When compared to the S-containing system 15, the desired ring expanded isomer is $9.4-11.9 \mathrm{kcal} \mathrm{mol}^{-1}$ more stable in the N/O-containing analogues 16-18. This clearly illustrates the significant additional challenge of accessing thiolactones using the SuRE method; for a more visual representation of the stark difference in the S-containing system 15, see the simplified potential energy surfaces depicted in Fig. 2.

Nonetheless, additional calculations on the higher homologues of sulfur-containing systems gave hope that the approach may still be feasible; for example, S-containing systems 19-21 were treated in the same way, and it was calculated that the ring expanded product is the lowest energy isomer for 8- and 9-membered ring starting materials, suggesting that these larger ringed variants are viable and have a higher chance of success. Therefore, it was decided to focus on larger ring starting materials, starting with readily available 8- and 13-membered ring lactams.

\section{Protecting group strategy selection}

A key decision was the choice of protecting group for the thiol. Three complementary strategies were explored, the first based on S-acetate protection. Thus, 8- and 13-membered lactams 21a and 21b were both reacted with acid chloride 22 using our 


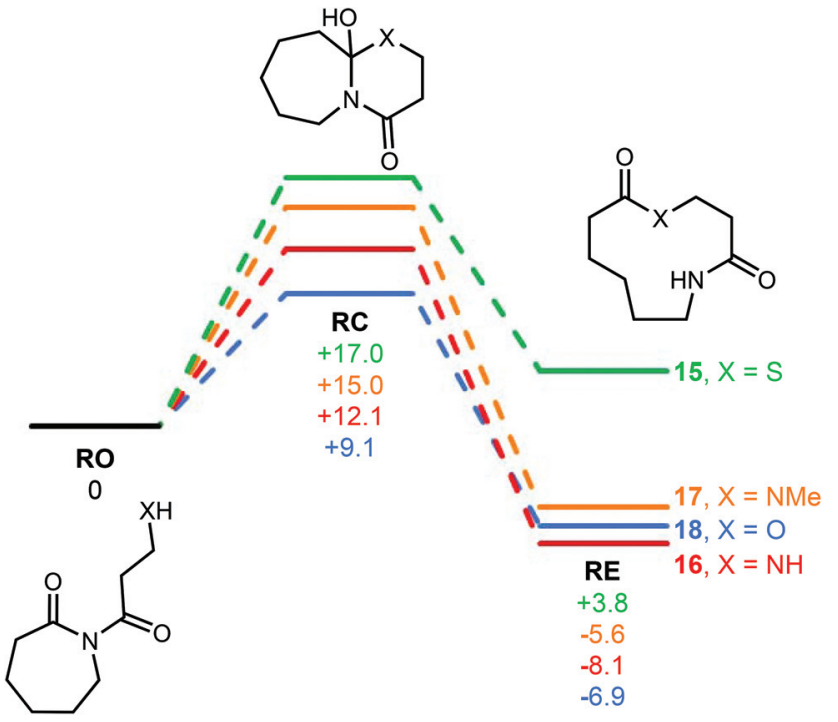

Fig. 2 Simplified potential energy surfaces for 15-18 calculated using a DFT/B3LYP/6-31G* approach. $\Delta G_{\text {rel }}^{\circ}$ values at $298 \mathrm{~K}$ are given in kcal $\mathrm{mol}^{-1}$. Note that this diagram depicts the calculated relative Gibbs free energies of the ground states of the three isomeric species in the ring expansion equilibrium only. Transition state energies were not calculated.

published $N$-acylation conditions to form imides 23a and 23b (Scheme 3). We then explored cleavage of the acetyl protecting group via the addition of various nucleophilic reagents, in the hope that this would result in concomitant ring expansion to form thiolactones. Of the conditions surveyed, stirring imides 23a and 23b with piperidine at RT in DCM were the most effective, forming thiolactones $\mathbf{2 0}_{\mathbf{R E}}$ and $\mathbf{2 4 b}$ in $29 \%$ and $36 \%$ yield respectively. However, chemoselectivity was a significant challenge using this approach; in particular, we were unable to fully discriminate between the three carbonyl groups of 23a. For example, unwanted reaction at the cyclic carbonyl C-3 (e.g. to form linear products 25-27) and/or unwanted C-2 attack (to form 28/29 and reform 21a) led to a reduction in yield for the desired product in all cases (see ESI, Table $\mathrm{S} 1 \uparrow$ for a full list of conditions trialled, reaction outcomes and synthetic details).

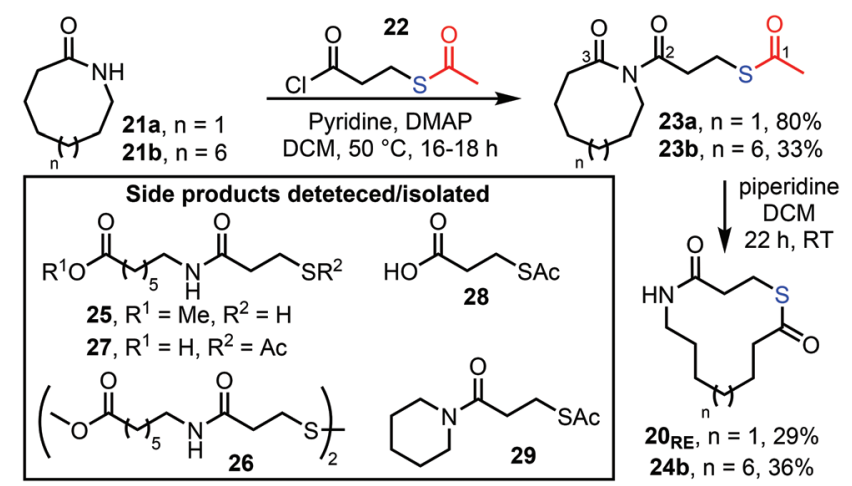

Scheme 3 SuRE using an S-Ac protecting group strategy.
With the S-Ac method proving difficult, we instead turned to an acid-labile trityl (Trt) protecting group strategy. ${ }^{20}$ Exploratory studies focused on 13-membered ring lactam 21b, which was converted into imide $\mathbf{3 1 b}$ via our standard method, using acid chloride 30. Various combinations of acids and trityl scavenger reagents were then trialled, to cleave the Trt protecting group and promote ring expansion into 24b (see ESI, Table S2 $\uparrow$ for a full list of conditions, reaction outcomes and synthetic details). The best conditions found were those summarised in Scheme 4, in which the Trt group was first cleaved using excess TFA in the presence of a silane scavenger reagent. This was then followed by aqueous workup and finally, stirring the product mixture overnight with an excess of DBU in DCM at RT, which promoted ring expansion to form the desired thiolactone product $\mathbf{2 4 b}$. The yield was muchimproved (56\%) compared to the S-Ac strategy, but side product formation was still not fully avoided, with a new condensation side product $\mathbf{3 3}$ observed under some of the conditions tested (likely as a result of the switch to acidic conditions), and thiol 32 was also isolated in some cases. ${ }^{21}$

Finally, a third protecting group strategy based on the use of the fluorenylmethyl (Fm) group was explored. The Fm protecting group is used relatively infrequently in organic synthesis (certainly compared to the related Fmoc protecting group) but it has attractive properties for the protections of thiols. ${ }^{22}$ The deprotection of Fm-protected thiols is typically done under conditions similar to those used to cleave Fmoc protecting groups from amines (e.g. using organic amine bases at RT). Given that such conditions were effective in our earlier work on SuRE using Fmoc-protected amino acid derivatives ${ }^{16 c, d}$ we reasoned that this approach might reduce side product formation in the present study. Thus, the $\mathrm{N}$-acylation of lactam 21b with S-Fm-containing acid chloride 34 was performed to form imide $\mathbf{3 5 b}$ (Scheme 5), which was taken directly onto the next step without purification. A nonnucleophilic base (DBU) was chosen to cleave the Fm protecting group, and pleasingly this promoted concomitant protecting group cleavage/ring expansion to form $\mathbf{2 4 b}$ in $54 \%$ yield over the overall 2 step telescoped sequence from $2 \mathbf{2 1 b}$. Frustratingly however, the product was accompanied by formation of another side product not observed in using the

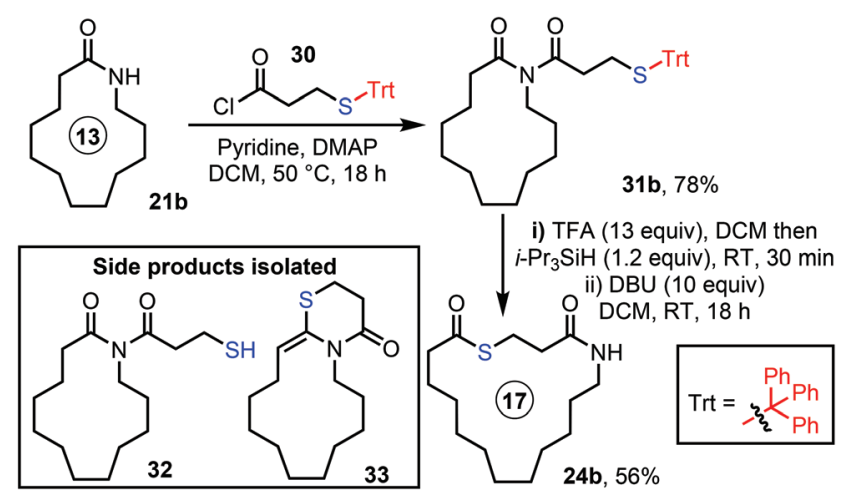

Scheme 4 SuRE using an S-Trt protecting group strategy. 

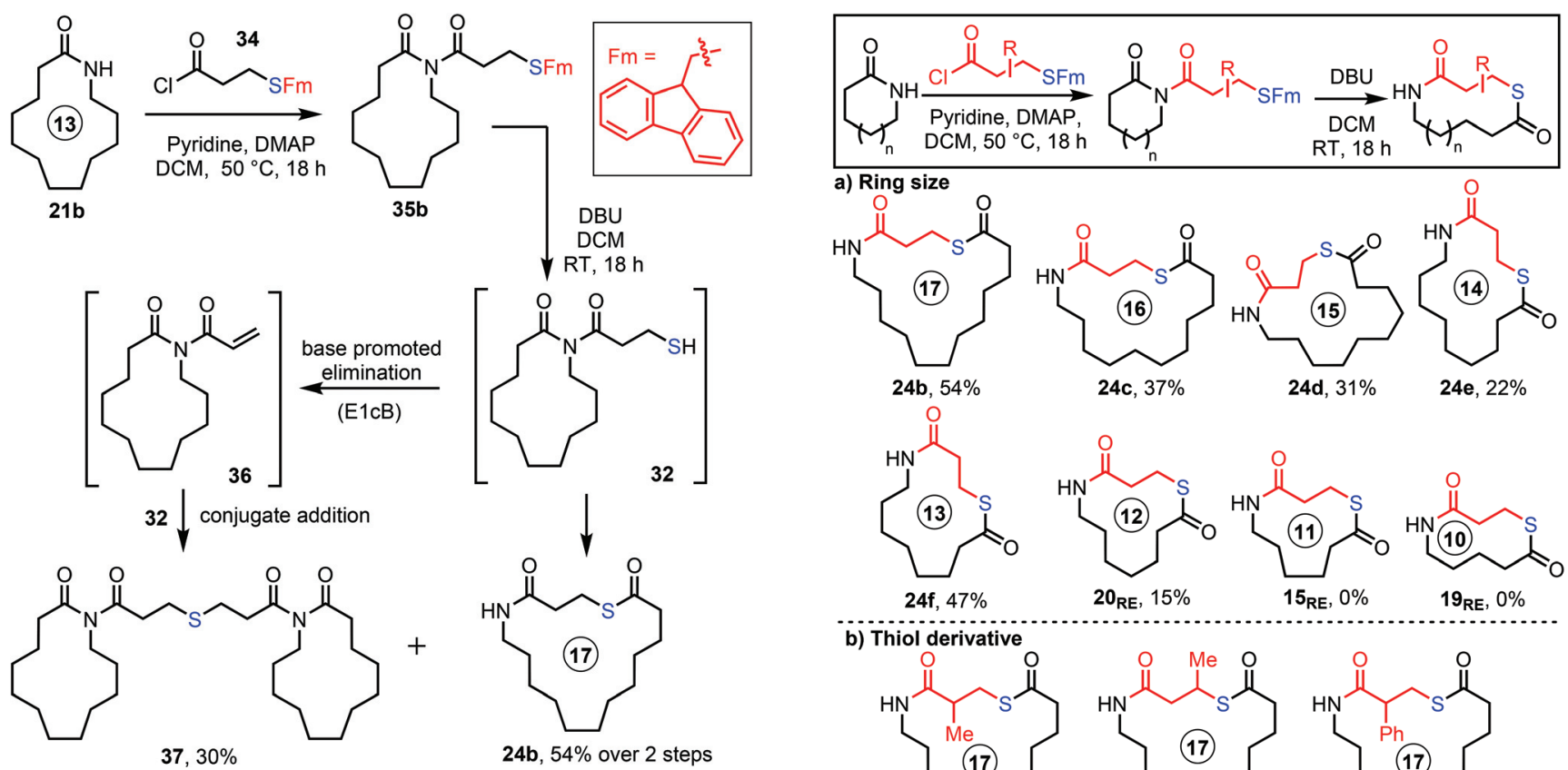

Scheme 5 SURE using an S-Fm protecting group strategy.

other protecting group strategies, in this case dimer 37. We presume that $\mathbf{3 7}$ is formed via base promoted elimination of 32 (or 35b) to form acrolein derivative 36 , which then reacts with another molecule of thiol 32 via conjugate addition.

\section{Reaction scope}

Despite not being able to fully suppress all side reactions, we were satisfied with the overall yield of $24 \mathrm{~b}$ ( $54 \%$ over 2 steps) achievable using the S-Fm protocol. Therefore, this approach was taken forward onto the reaction scoping phase of the project. We started by examining the effect of varying the lactam ring size, with 6-13-membered lactams all tested, using S-Fm-tethered acid chloride $\mathbf{3 4}$ and the procedure in Scheme 6. Pleasingly, the ring expanded thiolactone products were obtained for all the 8-13-membered parent lactams (24bf and $20_{\mathrm{RE}}, \mathbf{1 5 - 5 4 \%}$, Scheme 6a). Overall yields for the telescoped acylation/Fm cleavage/ring expansion sequence were generally modest, with the lowest yield obtained for the $8 \rightarrow$ 12-membered ring transformation to form $\mathbf{2 0} \mathbf{R E}$, which is not surprising given that the DFT results discussed earlier (Scheme 2) predicted this to be a borderline case thermodynamically. Conversely, the 10- and 11-membered ring products $15_{\mathbf{R E}}$ and $19_{\mathbf{R E}}$ were not formed at all, which was expected given that the ring opened imide isomers were calculated to be significantly lower in energy than the ringexpanded products for these examples.

Branching is tolerated on the linear fragment, with thiolactones $\mathbf{2 4 g}$-j all being formed using the standard protocol (Scheme 6b). The yields were comparatively low for the phenylsubstituted systems, likely due to competing elimination reactions; for example, in forming $\mathbf{2 4 j}$, a significant quantity of a cinnamyl side product (resulting from base-promoted

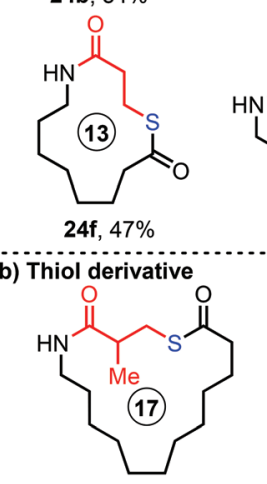

$24 \mathrm{~g}, 58 \%$
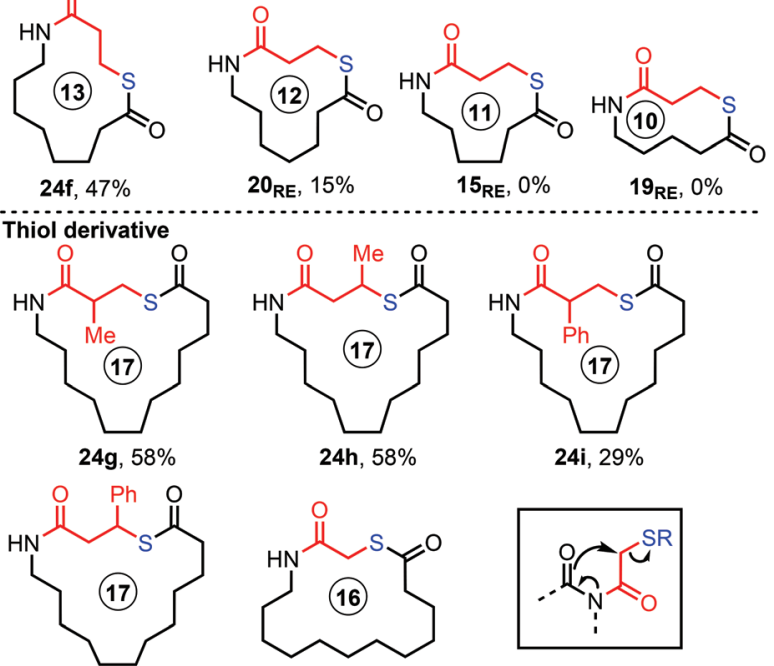

$20_{\text {RE }}, 15 \%$
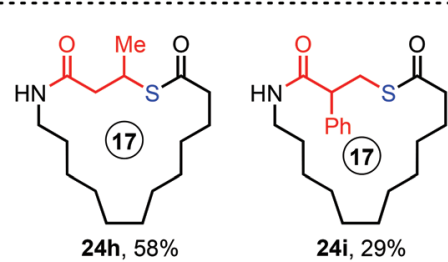

$24 \mathrm{j}, 8 \%{ }^{\mathrm{b}}$

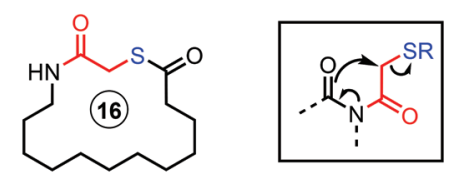

c) Succesive Ring Expansion ${ }^{\text {a }}$
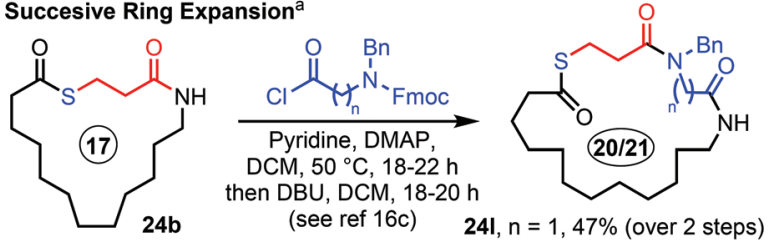

$\mathbf{2 4 m}, \mathrm{n}=2,14 \%$ (over 2 steps)

Scheme 6 Scope of the SuRE method using thiol-tethered carboxylic acid derivatives. ${ }^{a}$ Using our published method (ref. 16c). ${ }^{b} \mathrm{~N}$-Acylation step performed at RT (see ESI† for full details).

S-elimination, c.f. Scheme 5) was observed in the reaction mixtures, even upon reducing the temperature for the $N$-acylation step. ${ }^{23}$ Unfortunately, we were unable to form 16-membered thiolactone $24 \mathbf{k}$ in the same way, using a shorter $\alpha$-substituted homologue of the S-Fm-tethered acid chloride, with substrate degradation via a pathway known to lead to polymerisation (Scheme 6b box) proposed to be the main problem in this case. $^{24}$

We also confirmed that thiolactone-containing lactams are able to undergo further ring expansion via SuRE with amino acid chloride derivatives, with doubly expanded macrocycles 241 and $\mathbf{2 4 m}$ both prepared from 24b using our published method (Scheme 6c). The yields were lower than those of typical amino acid SuRE reactions, which is likely to be a consequence of the relatively reactive thiolactone enabling side reactions of the type already described, but nonetheless we 
were pleased to learn that thiolactones can be incorporated into larger macrocycles as part of iterative ring expansion sequences.

Other attempts to use the new method as part of longer sequences were more challenging however; for example, we were unable to form a doubly expanded product via the sequential insertion of two thiolactones (e.g. 40, Scheme 7) using the standard protocol. Thankfully, we were able to develop a reasonable work-around by using the S-Trt protecting group strategy and adding purification steps at intermediate points in the synthesis (Scheme 7). For example,

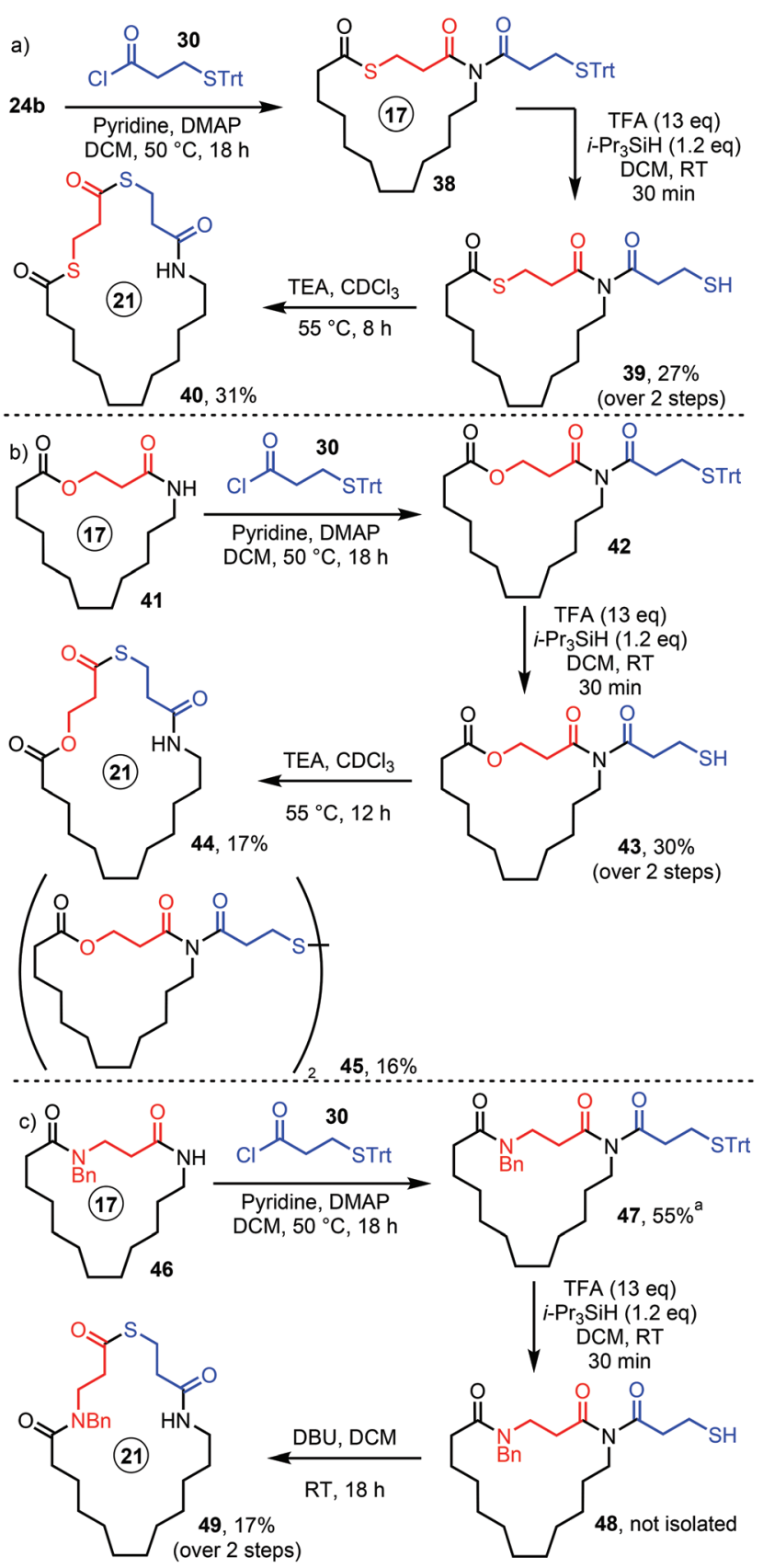

Scheme 7 Successive ring expansion- special cases. ${ }^{a}$ Contains trace impurities (see ESI $\dagger$ ).
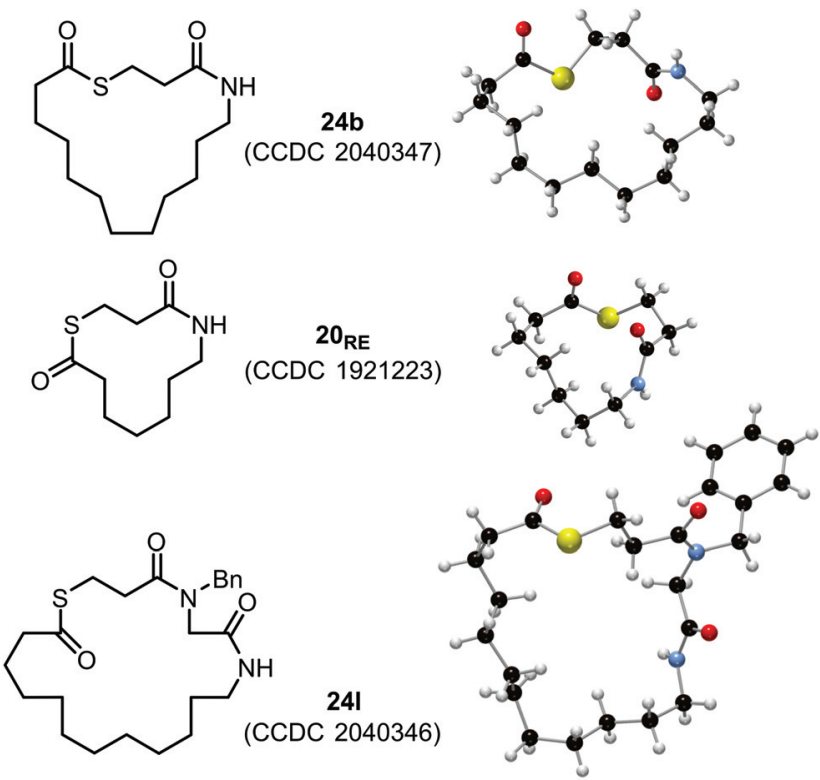

Fig. 3 X-ray crystal structures for $24 \mathrm{~b}, 20_{\mathrm{RE}}$ and $24 \mathrm{l}$.

$N$-acylation of thiolactone-containing lactam $24 \mathbf{b}$ with acid chloride 30, followed by cleavage of the Trt protecting group furnished imide thiol 39, with chromatographic purification used after each step. Triethylamine was then used to promote the ring expansion, which was done in $\mathrm{CDCl}_{3}$ to aid reaction monitoring, thus affording bis-thiolactone $\mathbf{4 0}$ in modest yield (Scheme 7a).

Macrocycle 44, a product which contains a lactam, a lactone, and a thiolactone group, was also prepared using a similar strategy, starting from a previously reported lactone SuRE product 41 (Scheme $7 \mathrm{~b}$ ). ${ }^{16 d}$ Another previously unobserved side product was isolated in this case, disulfide 45 , presumably as a result of oxidation of $\mathbf{4 3}$ by adventitious oxygen; incidentally, we believe that disulfide formation may also be a minor side reaction in other reactions featured in this manuscript, although this was the only case where it was confirmed via isolation of a pure product. Finally, macrocycle $\mathbf{4 9}$ was prepared, starting from bis-lactam $\mathbf{4 6} ;^{16 c}$ in this case, the ring expansion step was performed in DCM using DBU as the base (Scheme 7c).

To add additional support to the structural assignments made in this study, X-ray crystal structures were obtained for macrocyclic lactones $\mathbf{2 4 b}, \mathbf{2 0}$ RE and $24 \mathbf{l}$ (Fig. 3). ${ }^{25}$

\section{Conclusions}

Ascertaining whether thiol-derivatives can be used in SuRE reactions was a major reason for undertaking this study. In this manuscript we have shown that they can, despite the transformation being more challenging than previous SuRE variants; while yields of $>90 \%$ are common in our N- and O-SuRE processes, we have been unable to replicate this 
level of synthetic efficiency in the thiolactone-forming SuRE variants. The reduced thermodynamic driving force (supported by the DFT studies, see Scheme 2 and Fig. 2) for ring expansion is likely to be a significant factor that contributes to this difference; the DFT calculations as performed do not directly probe the reaction kinetics, but given the shift in calculated Gibbs free energy change for ring expansion in the S-containing cases, it is reasonable to predict that there would also be an associated increase in transition state energies (e.g. considering Hammond's postulate). ${ }^{26} \mathrm{~A}$ reduced reaction rate is not necessarily a problem when considered in isolation, but it is when kinetically accessible side reactions compete with the desired transformation, which we have clearly demonstrated to be the case for this system. ${ }^{27}$

More positively, thiolactone-forming ring expansion processes are rare in the literature, ${ }^{13,14}$ and we are pleased to learn that thiolactones can be incorporated into ring-expanded lactams using SuRE. Three complementary protecting group strategies have been explored and 15 novel macrocyclic thiolactones have been prepared using the new methods, which generally proceed in good overall yield (up to 58\%) over the telescoped $N$-acylation/protecting group cleavage/ring expansion sequence $(\approx 83 \%$ per transformation). Although we are happy to acknowledge that this study was frustrating at times, observing and appreciating the various unexpected side reactions encountered will certainly help to inform future work on SuRE, as well as related studies on side chain insertion ring expansion reactions.

\section{Experimental}

Full synthetic details and spectroscopic data for all compounds are provided in the ESI. $\dagger$ A general synthetic procedure for the S-fluorenylmethyl (S-Fm) method (including $\mathrm{N}$-acylation, S-Fm clevage and ring expansion) is provided here: a mixture of lactam $(0.5 \mathrm{mmol})$, DMAP $(0.05 \mathrm{mmol})$ and pyridine $(3.0 \mathrm{mmol})$ in DCM $(7 \mathrm{~mL})$ under an argon atmosphere was stirred at RT for $30 \mathrm{~min}$. Next, a solution of acid chloride $34(1.5 \mathrm{mmol})$ in DCM $(3 \mathrm{~mL})$ was added and the resulting mixture was heated at reflux $\left(50{ }^{\circ} \mathrm{C}\right.$ heating block temperature) for $18 \mathrm{~h}$. The mixture was then diluted with DCM $(10 \mathrm{~mL})$ and washed with $10 \%$ aq. $\mathrm{HCl}(10 \mathrm{~mL})$. The aqueous layer was then extracted with DCM $(3 \times 10 \mathrm{~mL})$ and the combined organic extracts dried over $\mathrm{MgSO}_{4}$ and concentrated in vacuo. The crude material was then re-dissolved in DCM $(10 \mathrm{~mL})$ and DBU $(5.00 \mathrm{mmol})$ was added, followed by stirring at RT for $18 \mathrm{~h}$, before the solvent was removed in vacuo and the ring-expanded product purified by flash column chromatography.

\section{Conflicts of interest}

There are no conflicts to declare.

\section{Acknowledgements}

The authors thank the EPSRC for the computational equipment used in this study (EP/H011455/1 and EP/ K031589/1) and the Department of Chemistry, University of York for the provision of an Eleanor Dodson Fellowship (to W. P. U.) and for supporting K. Y. P. and R. G. E. with PhD studentships. Thanks also go to Zhongzhen Yang for help with preliminary studies and to Sam Hart for X-ray crystallography.

\section{Notes and references}

1 For a review, see: F. Pietrocola, L. Galluzzi, J. M. Bravo-San Pedro, F. Madeo and G. Kroemer, Cell Metab., 2015, 21, 805.

2 (a) P. E. Dawson, T. W. Muir, I. Clark-Lewis and S. B. Kent, Science, 1994, 266, 776 For useful perspective on $\mathrm{N} \rightarrow \mathrm{S}$ acyl transfer in peptide thioester synthesis (conceptually related to the methodology described in this manuscript), see: (b) D. Macmillan, A. Adams and B. Premdjee, Isr. J. Chem., 2011, 51, 885.

3 (a) M. Sibrian-Vazquez, J. O. Escobedo, S. Lim, G. K. Samoei and R. M. Strongin, Proc. Natl. Acad. Sci. U. S. A., 2010, 107, 551; (b) L. F. Agnati, S. Ferré, S. Genedani, G. Leo, D. Guidolin, M. Filaferro, P. Carriba, V. Casadó, C. Lluis, R. Franco, A. S. Woods and K. Fuxe, J. Proteome Res., 2006, 5, 3077; (c) Y. Vallee, I. Shalayel, K.-D. Ly, K. V. R. Rao, G. de Paëpe, K. Märker and A. Milet, Int. J. Dev. Biol., 2017, 61, 471.

4 For a classical study comparing the rates of hydrolysis of lactones and thiolactones, see: C. M. Stevens and D. S. Tarbell, J. Org. Chem., 1954, 19, 1996.

5 (a) C.-L. J. Wang and J. M. Salvino, Tetrahedron Lett., 1984, 25, 5243; (b) D. V. Ferraris, P. Majer, C. Ni, C. E. Slusher, R. Rais, Y. Wu, K. M. Wozniak, J. Alt, C. Rojas, B. S. Slusher and T. Tsukamoto, J. Med. Chem., 2014, 57, 243; (c) D. H. Kim, C. J. Guinosso, G. C. Buzby Jr., D. R. Herbst, R. J. McCaully, T. C. Wicks and R. L. Wendt, J. Med. Chem., 1983, 26(3), 394.

6 For a review that includes a useful and detailed account of the synthesis and reactivity of thiolactones, see: (a) Z. Paryzek and I. Skiera, Org. Prep. Proced. Int., 2007, 39, 203 For selected more recent applications of thiolactones in synthetic chemistry, see: (b) J. Huang, F. Xiong, Z.-H. Wang and F.-E. Chen, Helv. Chim. Acta, 2009, 92, 1445; (c) D. Frank, P. Espeel, S. Classens, E. Mes and F. E. Du Prez, Tetrahedron, 2016, 72, 6616.

7 (a) P. Espeel and F. E. Du Prez, Eur. Polym., 2015, 62, 247; (b) H. R. Kricheldorf, S. M. Weidner and F. Scheliga, Polym. Chem., 2017, 8, 1589; (c) P. Espeel, F. Goethals and F. E. Du Prez, J. Am. Chem. Soc., 2011, 133, 1678; (d) S. Martens, A. Landuyt, P. Espeel, B. Devreese, P. Dawyndt and F. Du Prez, Nat. Commun., 2018, 9, 4451. 
8 (a) K. Steliou, P. Salama and J. Corriveau, J. Org. Chem., 1985, 50, 4969; (b) D. Bhar and S. Chandrasekaran, Tetrahedron, 1997, 53, 11835.

9 For papers on the importance of ring size in end-to-end cyclisation reactions of larger rings, see: (a) G. Illuminati and L. Mandolini, Acc. Chem. Res., 1981, 14, 95; (b) J. Fastrez, J. Phys. Chem., 1989, 93, 2635; (c) J. C. Collins and K. James, MedChemComm, 2012, 3, 1489; (d) H. Kurouchi and T. Ohwada, J. Org. Chem., 2020, 85, 876.

10 For general perspective on macrocycle synthesis, see: (a) E. Marsault and M. L. Peterson, J. Med. Chem., 2011, 54, 1961; (b) A. K. Yudin, Chem. Sci., 2015, 6, 30; (c) K. T. Mortensen, T. J. Osberger, T. A. King, H. F. Sore and D. R. Spring, Chem. Rev., 2019, 119, 10288; (d) Practical Medicinal Chemistry with Macrocycles, ed. E. Marsault and M. L. Peterson, Wiley, 2017; (e) S. D. Appavoo, S. Huh, D. B. Diaz and A. K. Yudin, Chem. Rev., 2019, 119, 9724; (f) I. V. Smolyar, A. K. Yudin and V. G. Nenajdenko, Chem. Rev., 2019, 119, 10032; (g) I. Saridakis, D. Kaiser and N. Maulide, ACS Cent. Sci., 2020, 6, 1869.

11 For reviews of ring expansion chemistry, see: (a) M. Hesse, in Ring Enlargement in Organic Chemistry, Wiley-VCH, Weinheim, 1991; (b) W. P. Unsworth and J. R. Donald, Chem. - Eur. J., 2017, 23, 8780; (c) K. Prantz and J. Mulzer, Chem. Rev., 2010, 110, 3741; (d) T. C. Stephens and W. P. Unsworth, Synlett, 2020, 31, 133; (e) A. K. Clarke and W. P. Unsworth, Chem. Sci., 2020, 11, 2876.

12 (a) L. Li, Z.-L. Li, F.-L. Wang, Z. Guo, Y.-F. Cheng, N. Wang, X.-W. Dong, C. Fang, J. Liu, C. Hou, B. Tan and X.-Y. Liu, Nat. Commun., 2016, 7, 13852; (b) J. E. Hall, J. V. Matlock, J. W. Ward and J. Clayden, Angew. Chem., Int. Ed., 2016, 55, 11153; (c) Z.-L. Li, X.-H. Li, N. Wang, N.-Y. Yang and X.-Y. Liu, Angew. Chem., Int. Ed., 2016, 55, 15100; (d) R. Mendoza-Sanchez, V. B. Corless, Q. N. N. Nguyen, M. Bergeron-Brlek, J. Frost, S. Adachi, D. J. Tantillo and A. K. Yudin, Chem. - Eur. J., 2017, 23, 13319; (e) R. Costil, Q. Lefebvre and J. Clayden, Angew. Chem., Int. Ed., 2017, 56, 14602; $(f)$ D. R. Loya, A. Jean, M. Cormier, C. Fressigné, S. Nejrotti, J. Blanchet, J. Maddaluno and M. De Paolis, Chem. - Eur. J., 2018, 24, 2080; (g) N. Wang, Q.-S. Gu, Z.-L. Li, Z. Li, Y.-L. Guo, Z. Guo and X.-Y. Liu, Angew. Chem., Int. Ed., 2018, 57, 14225; (h) Y. Zhou, Y.-L. Wei, J. Rodriguez and Y. Coquerel, Angew. Chem., Int. Ed., 2019, 58, 456; (i) E. Reutskaya, A. Osipyan, A. Sapegin, A. S. Novikov and M. Krasavin, J. Org. Chem., 2019, 84, 1693; (j) A. Lawer, J. A. Rossi-Ashton, T. C. Stephens, B. J. Challis, R. G. Epton, J. M. Lynam and W. P. Unsworth, Angew. Chem., Int. Ed., 2019, 58, 13942; (k) S. Grintsevich, A. Sapegin, E. Reutskaya, S. Peintner, M. Erdélyi and M. Krasavin, Eur. J. Org. Chem., 2020, 5664.

13 (a) H. C. De Araújo and J. R. Mahajan, Synthesis, 1978, 228;

(b) J. R. Mahajan and H. C. De Araújo, Synthesis, 1980, 64;

(c) R. Malherbe, G. Rist and D. Bellus, J. Org. Chem., 1982, 48, 860; (d) E. Vedejs, J. M. Dolphin and H. Mastalerz, J. Am. Chem. Soc., 1983, 105, 127; (e) C. Meng, J.-J. Li,
X.-M. Liang, J.-J. Zhang and D.-Q. Wang, Phosphorus, Sulfur Silicon Relat. Elem., 2014, 189, 1529.

14 Thiolactones are also involved in ring expansion cascade reactions (known as 'thia zip reactions'), although are intermediates and not the final products in these reactions, see: J. P. Tam, Y.-A. Lu and Q. Yu, J. Am. Chem. Soc., 1999, 121, 4316.

15 (a) Y. Nakashita and M. Hesse, Angew. Chem., 1981, 93, 1077; (b) S. Stanchev and M. Hesse, Helv. Chim. Acta, 1989, $72,1052$.

16 (a) C. Kitsiou, J. J. Hindes, P. I'Anson, P. Jackson, T. C. Wilson, E. K. Daly, H. R. Felstead, P. Hearnshaw and W. P. Unsworth, Angew. Chem., Int. Ed., 2015, 54, 15794; (b) L. G. Baud, M. A. Manning, H. L. Arkless, T. C. Stephens and W. P. Unsworth, Chem. - Eur. J., 2017, 23, 2225; (c) T. C. Stephens, M. Lodi, A. Steer, Y. Lin, M. Gill and W. P. Unsworth, Chem. - Eur. J., 2017, 23, 13314; (d) T. C. Stephens, A. Lawer, T. French and W. P. Unsworth, Chem. - Eur. J., 2018, 24, 13947 For the application of SuRE reactions in the work of another group, see: $(e) \mathrm{C}$. Zhao, Z. Ye, Z.-X. Ma, S. A. Wildman, S. A. Blaszczyk, L. Hu, I. A. Guizei and W. Tang, Nat. Commun., 2019, 10, 4015, DOI: 10.1038/s41467-019-11976-2.

17 This method was introduced by our group in ref. $16 d$, and more fully explored and benchmarked against other computational methods in: A. Lawer, R. G. Epton, T. C. Stephens, K. Y. Palate, M. Lodi, E. Marotte, K. J. Lamb, J. K. Sangha, J. Lynam and W. P. Unsworth, Chem. - Eur. J., 2020, 26, 12674 See ESI for full details of the new computational chemistry results reported in this paper. $\dagger$

18 For computational chemistry studies on related ring expansion processes, see ref. $12 d$ and $i$.

19 A guideline established in our earlier work (ref. 17) is that the ring expanded isomer should be the most stable by $>3 \mathrm{kcal} \mathrm{mol}^{-1}$ for ring expansion to be the predicted outcome.

20 P. Majer, P. F. Jackson, G. Delahanty, B. S. Grella, Y.-S. Ko, W. Li, Q. Liu, K. M. Maclin, J. Poláková, K. A. Shaffer, D. Stoermer, D. Vitharana, E. Y. Wang, A. Zakrzewski, C. Rojas, B. S. Slusher, K. M. Wozniak, E. Burak, T. Limsakun and T. Tsukamoto, J. Med. Chem., 2003, 46, 1989.

21 Another important consideration when using this approach is that in our hands, the $N$-acylation step (i.e. $\mathbf{2 1 b} \rightarrow \mathbf{3 1 b}$ was somewhat capricious, which we think is a consequence of the requirement to form an acid chloride in the presence of an acid labile protecting group. Efficient $N$-acylation can still be achieved by forming and reacting acid chloride $\mathbf{3 0}$ quickly, but compared with other SuRE variants, this is an additional complication.

22 (a) M. Ruiz-Gayo, F. Albericio, E. Pedroso and E. J. Giralt, Chem. Soc., Chem. Commun., 1986, 1501; (b) E. J. Corey, D. Y. Gin and R. S. Kania, J. Am. Chem. Soc., 1996, 118, 9202; (c) B. Ponsati, E. Giralt and D. Andreu, Tetrahedron, 1990, 46, 8255 . 
23 Under the standard conditions, the following cinnamyl imide product was obtained and isolated in 70\% yield (see ESI compound S6): $\dagger$

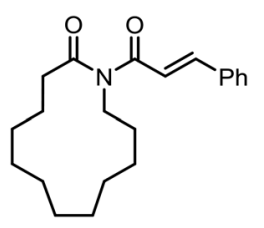

24 (a) D. R. Moore and L. J. Mathias, J. Org. Chem., 1987, 52, 1599; (b) L. J. Mathias and D. R. Moore, J. Am. Chem. Soc., 1985, 107, 5817.

25 CCDC 2040347 (24b), 1921223 (20 RE $_{\text {) }}$ and 2040346 (24l) contain the crystallographic data for these macrocyclic thiolactones, see: $\dagger$.
26 This is also supported qualitatively by the observation that free-thiol containing imides (e.g., 20 $\mathbf{R O}_{\mathbf{R O}}, 32,39$, and 43) were found to be isolable, kinetically stable species, while the analogous amine containing systems cannot be isolated as they ring expand spontaneously at RT (see ref. $16 c)$.

27 In total, six mechanistically distinct side reactions were observed during this study: (1) nucleophilic attack at the C-3 carbonyl leading to lactam opening (e.g., 25-27); (2) nucleophilic attack at the $\mathrm{C}-2$ carbonyl leading to cleavage of the external imide $\mathrm{C}-\mathrm{N}$ bond (e.g. 28, 29); (3) condensation (e.g. 33); (4) $\beta$-elimination of $\mathrm{S}$ (e.g. Scheme 5 and $\mathbf{2 4 \mathbf { j }}$ ); (5) dimerisation via conjugate addition (e.g. Scheme 5); (6) disulfide formation (e.g. 45, Scheme 7). 